\title{
Fast marching method and modified features fusion in enhanced dynamic hand gesture segmentation and detection method under complicated background
}

\begin{abstract}
Recent development in the field of human-computer interaction has led renewed interest in dynamic hand gesture segmentation based on gesture recognition system. Despite its long clinical success, dynamic hand gesture segmentation using webcam vision becomes technically challenging and suffers the problem of non-accurate and poor hand gesture segmentation where the hand region is not integral due to complicated environment, partial occlusion and light effects. Therefore, for segmenting complete hand gesture region and improving the segmentation accuracy, this study proposes a combination of four modified visual features segmentation procedures, which are skin, motion, skin moving as well as contour features and fast marching method. Quantitative measurement was performed for evaluating hand gesture segmentation algorithm. Besides, qualitative measurement was done to conduct a comparison based on segmentation accuracy with previous studies. Consequently, the experiment results showed a great enhancement in hand area segmentation with a high accuracy rate of $98 \%$.
\end{abstract}

Keyword: Hand gesture segmentation; Skin segmentation; Motion segmentation; Fast marching method; Entropy filter; Viola Jones; Contour segmentation 